\title{
STROMAL CHANGES IN INVASIVE CARCINOMA OF BREAST
}

\author{
Chasma $K^{1}$, Sankar $S^{2}$
}

1 Junior Resident, Department of Pathology, Government Medical College, Kottayam, Kerala, India. 2Professor and HOD, Department of Pathology, Government Medical College, Kottayam, Kerala, India.

\section{BACKGROUND}

ABSTRACT

Breast cancer is the most common malignancy in women worldwide. The current diagnostic and management practices in breast cancer takes into consideration the neoplastic cells only and the tumour stroma is not addressed properly. Recent studies have shown that tumour stroma play an important role in tumourigenesis, its behaviour and response to therapy. In this study, we are trying to describe the histopathological changes in tumour stroma with respect to tumour stroma ratio, microvessel density and tumour associated macrophages.

Aims and Objectives- To describe the histopathological changes in invasive carcinoma breast with respect to tumour stroma ratio (TSR), tumour associated macrophages (TAMs) and angiogenesis (Microvessel Density, MVD)

Settings- Department of Pathology, Government Medical College, Kottayam.

Design- Descriptive study.

\section{MATERIALS AND METHODS}

Study was conducted in first 134 biopsy specimens of invasive carcinoma breast received in the Department of Pathology, during the study period of 16 months (June 2017 to September 2018).

Analysis- SPSS software.

\section{RESULTS}

In the present study, $66.4 \%$ cases were of stroma low (stromal density $<50 \%$ ) type and $33.6 \%$ cases were of stroma high type (Stromal density $\geq 50 \%$ ), comparable with the study conducted by A M Moorman et al. $59 \%$ were found to have low microvessel density (MVD) and $41 \%$ high MVD. Equal proportion of cases were found in TAM low and TAM high group. The association was found to be statistically significant between stromal density and microvessel density ( $p$ value 0.042), and also between MVD and TAM ( $p$ value of 0.022 ).

\section{CONCLUSION}

No statistically significant association was detected between age, tumour grade, stage of the tumour, lymph node metastasis, or molecular subtypes with stromal density, microvessel density and tumour associated macrophage density. Statistically signific ant association was found between microvessel density with stromal density and tumour associated macrophages.

\section{KEY WORDS}

Invasive Carcinoma Breast, Stromal Density, Tumour Associated Macrophages, Microvessel Density

HOW TO CITE THIS ARTICLE: Chasma K, Sankar S. Stromal changes in invasive carcinoma of breast. J. Evolution Med. Dent. Sci. 2019;8(10):679-684, DOI: 10.14260/jemds/2019/151

\section{BACKGROUND}

Breast cancer is the most common non-skin malignancy in women and is a leading cause of cancer death worldwide. With an annual incidence of approximately 144, 000 new cases in India, breast cancer has now become the most common female cancer in urban India 1 . Breast cancer is rare in women younger than 25 years and its incidence increases rapidly after 30 years of age. The most common type is invasive ductal carcinoma. ${ }^{2}$

One third of cases of invasive carcinoma are classified into special morphologic type but the majority of breast cancers have no special type of characteristics and are termed invasive ductal carcinoma of no special type, or not otherwise specified (NOS/NST). ${ }^{3}$

'Financial or Other Competing Interest': None.

Submission 27-12-2018, Peer Review 22-02-2019,

Acceptance 28-02-2019, Published 11-03-2019.

Corresponding Author:

Chasma K,

Ushass, P. O, Kanhirode,

Kannur-670592, Kerala, India.

E-mail: chasmakelambeth@gmail.com

DOI: $10.14260 /$ jemds $/ 2019 / 151$

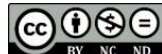

According to Nottingham Grading System, breast carcinoma is graded based on tubule formation, nuclear pleomorphism and mitotic rate. The outcome for women with breast cancer depends on the biologic features of carcinoma (Molecular or histologic type) and the extent to which cancer has spread (Stage) at the time of diagnosis. ${ }^{4}$ But in clinical practice sometimes the carcinoma behaves unexpectedly in spite of its low-grade status and favourable histology.

Recent studies have shown that the tumour microenvironment composed of non- cancer cells and their stroma play an important role in tumorigenesis, influencing the growth of cancer, determining its metastatic potential and possibly determining location of metastasis and thus impacting outcome of therapy. Though stromal cells are themselves non-malignant, their role in supporting cancer cell growth is so vital to the survival of the tumour that they have become an attractive target for chemotherapeutic agents. The mammary stroma consists of a compilation of cells, including fibroblasts/myofibroblasts, vascular, epithelial, smooth muscle, fat cells and cells of immune system. ${ }^{5}$ The immune cells of stroma are macrophages/ monocytes, neutrophils and lymphocytes. Monocytes are actively recruited into tumours and once in the tumour, they 
differentiate into tumour associated macrophages (TAM). ${ }^{5}$ The activated macrophages can either be M1 or M2 macrophages. CD 68 is a pan- macrophage marker which recognizes both M1 and M2 macrophages. ${ }^{6} \mathrm{CD} 163$ is a highly specific monocyte/ macrophage marker for M2 macrophages. ${ }^{7}$

Tumours require the formation of complex vascular network to meet the metabolic and nutritional needs for its growth. Tumour vessels formed are abnormal, non uniformly distributed, irregularly shaped, inappropriately branched and tortuous, often ending blindly. CD 34, CD 133, VEGFR -2 are positive markers for vascular cells. ${ }^{5}$

The aim of this study is to describe the histopathological changes in stroma in invasive carcinoma of breast with relevance to Tumour - Stromal Ratio (TSR), Tumour Associated Macrophages (TAM) and Angiogenesis (Micro Vessel Density MVD)

\section{MATERIALS AND METHODS}

\section{Study Design}

Descriptive study.

\section{Period of Study}

16 months, June 2017 to September 2018.

\section{Study Setting}

Department of Pathology, Govt. Medical College, Kottayam.

\section{Study Population}

All biopsied specimens of invasive carcinoma breast received in Department of Pathology, Govt. Medical College, Kottayam during the study period.

\section{Sample Size 8}

Sample size $N=\underline{4 p q}$

$\mathrm{d}^{2}$

$\mathrm{p}=$ prevalence $/$ proportion in previous study.

$q=100-p$

$\mathrm{d}=$ precision/ allowable error.

In the study by Moorman et al, proportion of patients classified as stroma high were found to be $40 \%$, and fixing the relative precision as $20 \%$ of $\mathrm{P}$, sample size $\mathrm{N}$ is calculated as 150.

\section{Study Tools}

1. Instruments to take bits of tissues to be studied.

2. Reagents for tissue processing.

3. Instruments for making paraffin blocks and cutting thin sections from it.

4. Glass slides and cover slips for mounting.

5. Microscope

6. Eosin- Haematoxylin staining.

7. Antibody and other reagents for immunohistochemical studies.

8. Proforma to record serial number, Biopsy number, Name, age, parity, clinical features, gross, histopathology and immunohistochemical features.

\section{Inclusion Criteria}

Histopathologically diagnosed cases of invasive carcinoma breast NOS will be included.

\section{Exclusion Criteria}

Cases without proper data, recurrent/ previously treated cases of invasive carcinoma breast, post neoadjuvant chemotherapy cases, special types of invasive carcinoma and male breast carcinoma cases will be excluded.

\section{Study Procedure}

Clinical details of each case were recorded first. Gross examination of the specimen was done. Tumour size measured in three dimensions and the largest dimension taken into account. Appropriate bits of tissues representative of areas to be studied were taken. All specimens will be fixed in formalin processed and embedded in paraffin.

4 microns thick sections were stained with Haematoxylin \& Eosin for routine histological examination. The amount of stroma was quantified after using scanner lens to select the most invasive part of the tumour and then using the $10 \mathrm{x}$ objective to score. Only fields where both stroma and tumour cells are present were selected for scoring. Visual assessment of stromal density to tumour is done for three different fields and average was taken. Labomed Lx 300 microscope was used to examine the slides. A cut- off of $50 \%$ was taken based on previous studies and Stroma - low defined as $<50 \%$ stroma and stroma - high as $\geq 50 \%$ stroma.

For immunohistochemistry 3-micron thick sections were cut and stained with Monoclonal Mouse Anti-human CD34, class II clone, QBend-10 antibody. Microvessel Density (MVD) was evaluated by counting anti - CD34 positive microvessels using light microscope and calculated by counting method developed by Weidner. After scanning the whole tumour area with light microscope under low magnification, the area with highest number of microvessels was identified as "hot spots" and microvessel were counted under high power (40 X). Any brown- stained single endothelial cell or endothelial cell clusters separated from surrounding tumour cells and connective tissue elements are considered to be a microvessel regardless whether they had a lumen or not. No erythrocyte was necessarily required in the lumen. Branching vessel structures are counted as a single vessel. Areas of necrosis are not considered while looking for microvessel density. Three different areas were counted, and an average is taken as the final value. After determining MVD for all patients the average is calculated and set as cut - off value to classify patients as low MVD and high MVD.

To quantify Tumour Associated Macrophages (TAM), slides were stained with CD68 antibody. We used KP-1 Mouse Monoclonal antibody. The slides were examined under scanner to identify areas with highest levels of TAM infiltration similar to microvessel counting. For each case, three hot spots in high- power field (40x) are selected for counting TAMs. Intratumoral TAMs are defined as macrophages within tumour cells and in direct contact with tumour cells, and stromal TAMs are defined as macrophages infiltrated in the tumour stroma of the invasive carcinoma. After counting, three different areas, the average numbers of total TAMs per high power field were calculated. A cut off value is found from mean of the total TAMs for assigning tumours into low and high TAM group. 


\section{Data Management and Analysis}

The data was entered in Microsoft excel and further statistical analysis was done using SPSS software (Version 24).

\section{The Statistical Methods used were-}

1. Mean, frequency and proportion for

- Age.

- Laterality.

- Quadrant involved.

- $\quad$ Stage at diagnosis.

- Nodal status at diagnosis.

- BR grade at diagnosis.

- Molecular subtype of cancer.

- Stromal density.

- Microvessel density.

- Tumour associated macrophages.

2. Strength of association between stromal density, microvessel density, and tumour associated macrophages with tumour stage, grade, nodal status, molecular subtype of cancer, were calculated using Chi- Square Test

\section{Consent}

This study was conducted on the specimens of Carcinoma breast, received in the Department of Pathology. Hence implied consent is present.

\section{RESULTS}

The present study was conducted in 134 cases of breast carcinoma specimens $(n=134)$ received in the Department of Pathology, Government Medical college, Kottayam during the study period of 16 months (June 2017 to September 2018). All the specimens were from female subjects.

The mean age of presentation of cases were found to be $54.21 \pm 11.62$ years. Majority of the cases were within the age group 41 - 50 years (35.1\%). Most of the lesions were right sided ones. Based on 2012 WHO TNM classification of tumours of breast 5 most cases were of stage II A (44 cases, $32.8 \%$ ) and grade II (81 cases, $60.4 \%$ ). In the present study, majority of cases (57 cases, $42.5 \%$ ) were having either no lymph node metastasis at diagnosis or there was no lymph node sampling done during surgery. 45 cases (33.6\%) had 13 axillary lymph nodes metastasis, 20 cases $(14.9 \%)$ had $4-9$ lymph node metastasis and 12 cases $(9 \%)$ had $\geq 10$ positive lymph nodes at the time of diagnosis. In the present study 50 $\%$ of the cases (67 cases) were of luminal A type, $26.9 \%$ (36 cases) were of Luminal B type, $13.4 \%$ cases (18 cases) were basal -like (Triple negative) and $9.7 \%$ cases (13 cases) were Her2 enriched type.

Assessed by light microscopic examination of $\mathrm{H}$ \& $\mathrm{E}$ stained slides, $66.4 \%$ cases (89 cases) were found to be stroma low (Stromal density < $50 \%$ ) type and $33.6 \%$ cases (45 cases) were of stroma high type. (Stromal density $\geq$ $50 \%)$.

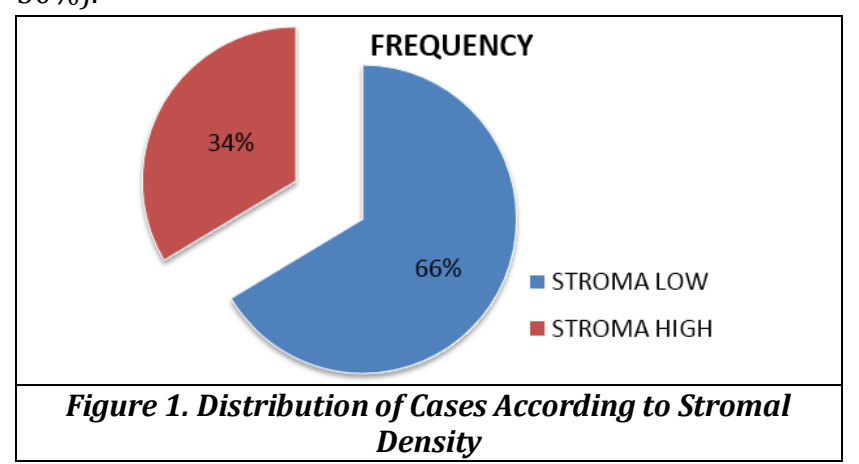

As assessed by anti CD34 staining and counting by Weidner count method, the mean value of microvessel density was found to be $30.93 \pm 13.565 .79$ cases (59\%) were found to have low microvessel density (MVD) and 55 cases (41\%) have high MVD.

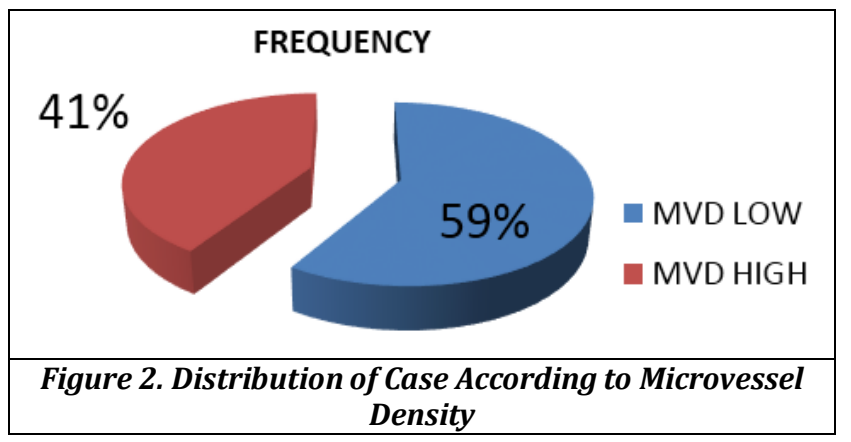

Assessed by anti CD68 staining and counting by Weidner count method the mean value of Tumour Associated Macrophage (TAMs) distribution was found to be $102.21 \pm$ 26.448 and equal proportion of cases were found to be within TAM low and TAM high group (67 cases).

In all age groups, cases with low stromal density and low microvessel density predominates. Whereas in the case of TAM infiltration, cases with high TAM predominates in most of the age groups. But no statistically significant association was found between these factors and age group.

\begin{tabular}{|c|c|c|c|c|c|c|}
\hline \multirow{2}{*}{$\begin{array}{c}\text { Age Group } \\
\text { (Years) }\end{array}$} & \multicolumn{2}{|c|}{$\begin{array}{c}\text { Stromal } \\
\text { Density }\end{array}$} & \multicolumn{2}{c|}{ MVD } & \multicolumn{2}{c|}{ TAM } \\
\cline { 2 - 7 } & Low & High & Low & High & Low & High \\
\hline $21-30$ & 0 & 1 & 1 & 0 & 1 & 0 \\
\hline $31-40$ & 9 & 3 & 8 & 4 & 11 & 1 \\
\hline $41-50$ & 32 & 15 & 28 & 19 & 22 & 25 \\
\hline $51-60$ & 22 & 16 & 22 & 16 & 17 & 21 \\
\hline $61-70$ & 19 & 4 & 11 & 12 & 10 & 13 \\
\hline $71-80$ & 4 & 6 & 6 & 4 & 4 & 6 \\
\hline $81-90$ & 3 & 0 & 3 & 0 & 2 & 1 \\
\hline
\end{tabular}

Table 1. Distribution of Stromal Density, MVD and TAM in Various Age Groups

\begin{tabular}{|c|c|c|c|c|c|c|}
\hline \multirow{2}{*}{$\begin{array}{c}\text { Tumour } \\
\text { Grade }\end{array}$} & \multicolumn{2}{|c|}{$\begin{array}{c}\text { Stromal } \\
\text { density }\end{array}$} & \multicolumn{2}{c|}{ MVD } & \multicolumn{2}{c|}{ TAM } \\
\cline { 2 - 7 } & Low & High & Low & High & Low & High \\
\hline BR Grade I & 21 & 13 & 22 & 12 & 19 & 15 \\
\hline BR Grade II & 54 & 27 & 48 & 33 & 41 & 40 \\
\hline $\begin{array}{c}\text { BR Grade } \\
\text { III }\end{array}$ & 14 & 5 & 9 & 10 & 7 & 12 \\
\hline \multicolumn{7}{|c|}{ Table 2. Distribution of Stromal Density, MVD \& TAM } \\
According to Tumour Grade \\
\hline
\end{tabular}

Proportion of cases with high MVD and high TAM were found to increase as tumour grade increased. Whereas in case of stromal density it was found to decrease with increase in tumour grade. The association between stromal density $(\mathrm{p}=$ 0.676) MVD (0.467) and TAM ( $p=0.407)$ with tumour grade were not found to be statistically significant. 


\begin{tabular}{|c|c|c|c|c|c|c|}
\hline \multirow{2}{*}{$\begin{array}{c}\text { Tumour } \\
\text { Stage }\end{array}$} & $\begin{array}{c}\text { Stromal } \\
\text { density }\end{array}$ & \multicolumn{2}{c|}{ MVD } & \multicolumn{2}{|c|}{ TAM } \\
\cline { 2 - 7 } & Low & High & Low & High & Low & High \\
\hline Stage IA & 13 & 6 & 13 & 6 & 9 & 10 \\
\hline Stage II A & 26 & 18 & 27 & 17 & 21 & 23 \\
\hline Stage II B & 22 & 4 & 13 & 13 & 12 & 14 \\
\hline Stage III A & 12 & 11 & 12 & 11 & 13 & 10 \\
\hline Stage III B & 7 & 3 & 7 & 3 & 6 & 4 \\
\hline Stage III C & 9 & 3 & 7 & 5 & 6 & 6 \\
\hline Table 3. Distribution of Stromal Density, MVD, TAM Based \\
on Tumour Stage \\
\hline
\end{tabular}

Cases with low stromal density and low microvessel density were found to be high in all stages of carcinoma but, in case of tumour associated macrophage infiltration no such pattern was observed. The association between tumour stage with stromal density $(\mathrm{p}=0.186)$, microvessel density $(\mathrm{p}=0.759)$, and tumour associated macrophage $(\mathrm{p}=0.955)$ showed no statistical significance.

\begin{tabular}{|c|c|c|c|c|c|c|}
\hline \multirow{2}{*}{$\begin{array}{c}\text { Node } \\
\text { Metastasis }\end{array}$} & \multicolumn{2}{|c|}{$\begin{array}{l}\text { Stromal } \\
\text { Density }\end{array}$} & \multicolumn{2}{|c|}{ MVD } & \multicolumn{2}{|c|}{ TAM } \\
\hline & Low & High & Low & High & Low & High \\
\hline $\mathrm{No} / \mathrm{Nx}$ & 39 & 18 & 36 & 21 & 29 & 28 \\
\hline N 1 & 30 & 15 & 24 & 21 & 20 & 25 \\
\hline N 2 & 11 & 9 & 12 & 8 & 12 & 8 \\
\hline N 3 & 9 & 3 & 7 & 5 & 6 & 6 \\
\hline \multicolumn{7}{|c|}{$\begin{array}{l}\text { Table 4. Distribution of Stromal Density, MVD, TAM Based } \\
\text { on Nodal Metastasis }\end{array}$} \\
\hline betwee & o stati & ically s & nificon & & & $\begin{array}{l}\text { ound } \\
\text { TAM }\end{array}$ \\
\hline
\end{tabular}

\begin{tabular}{|c|c|c|c|c|c|c|}
\hline \multirow{2}{*}{$\begin{array}{c}\text { Molecular } \\
\text { Subtype }\end{array}$} & \multicolumn{2}{|c|}{$\begin{array}{c}\text { Stromal } \\
\text { Density }\end{array}$} & \multicolumn{2}{c|}{ MVD } & \multicolumn{2}{|c|}{ TAM } \\
\cline { 2 - 7 } & Low & High & Low & High & Low & High \\
\hline Luminal A & 44 & 23 & 37 & 30 & 36 & 31 \\
\hline Luminal B & 23 & 13 & 26 & 10 & 17 & 19 \\
\hline $\begin{array}{c}\text { Her 2 } \\
\text { enriched }\end{array}$ & 12 & 1 & 4 & 9 & 7 & 6 \\
\hline Basal like & 10 & 8 & 12 & 6 & 7 & 11 \\
\hline
\end{tabular}

Table 5. Distribution of Stromal Density, MVD, TAM with Molecular Subtype of Carcinoma Breast

No statistically significant association was found between stromal density ( $p=0.173$ ), MVD (0.052) and TAM $(\mathrm{p}=0.052)$ with molecular subtype of carcinoma.

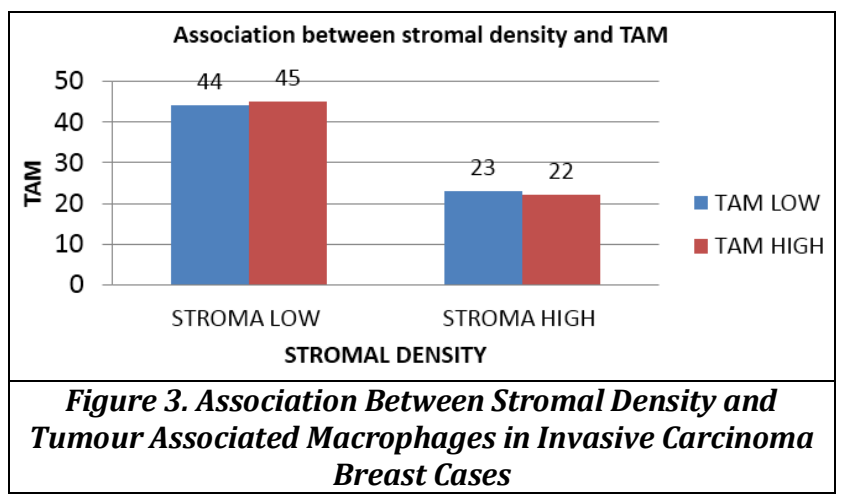

$49.4 \%$ (44 cases) of stroma low cases and $51.1 \%$ (23 cases) of stroma high cases are also found to have low tumour associated macrophage density (TAM). The $p$ value of association between stromal density and TAMs is found to be 0.855 , which statistically not significant.

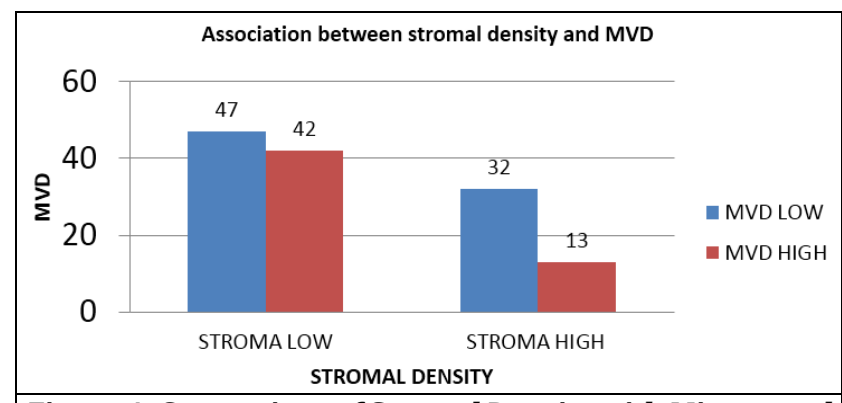

Figure 4. Comparison of Stromal Density with Microvessel Density

In the present study, $52.8 \%$ (47 cases) of low stromal density cases and $71.1 \%$ (32 cases) of high stromal density cases have low microvessel density. The $p$ value of association between stromal density and MVD is found to be 0.042 , which is statistically significant.

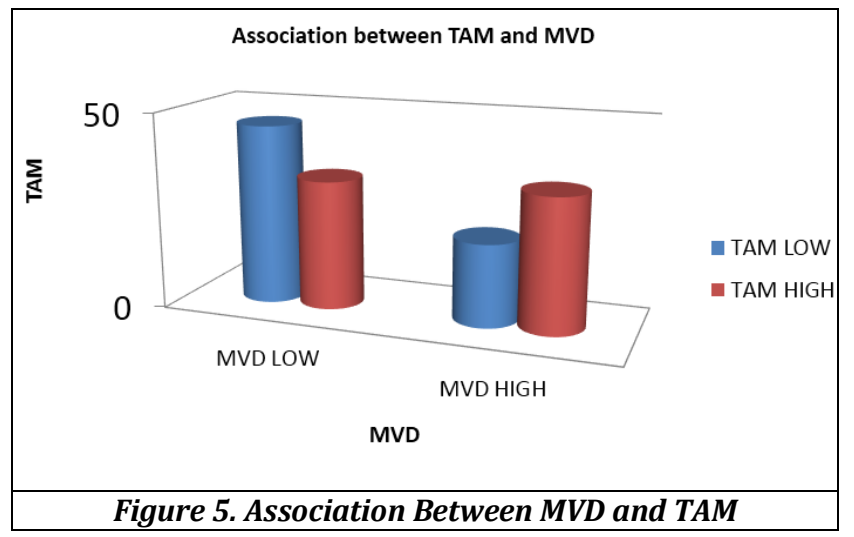

$58.2 \%$ of cases with low MVD had low TAM also. $61.8 \%$ of cases with high MVD had high TAM. The $p$ value of association was found to be $0.022(\mathrm{p}<0.05)$ and is statistically significant.

\section{Photomicrographs}

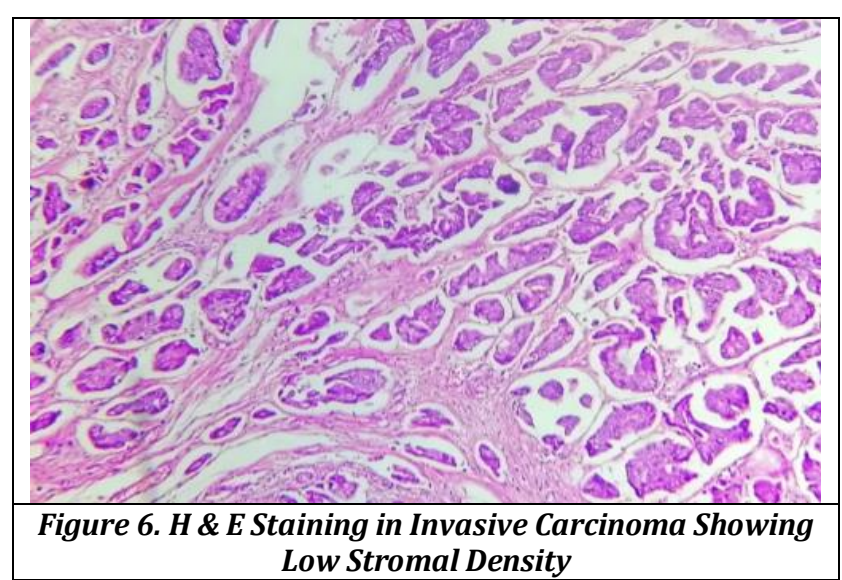



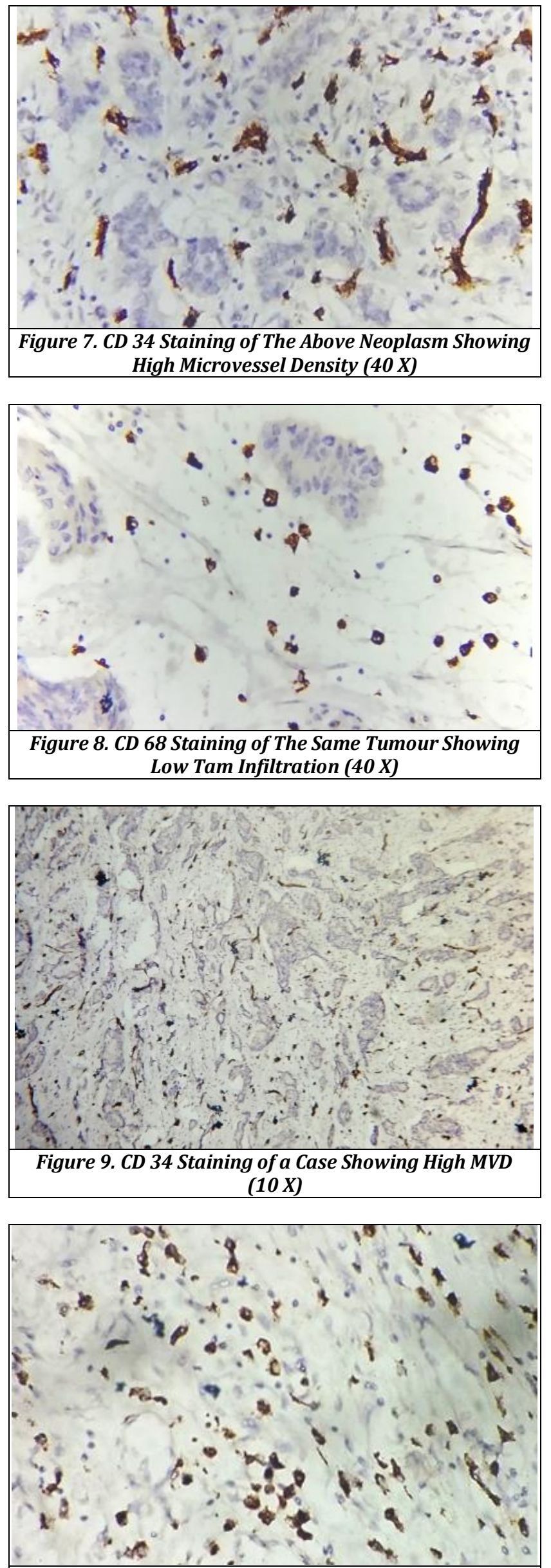

Figure 10. CD 68 Staining of the Same Case Showing High TAM Infiltration (40 X)

\section{DISCUSSION}

The mean age of the study population was $54.21 \pm 11.62$ years. This was comparable with study conducted by Chopra et al, which showed a mean age of $50.1 \pm 10.5$ years.

In the present study $66.4 \%$ of cases $(n=89)$ were found to be having low stromal density and $33.6 \%$ of cases $(n=45)$ having high stromal density. This was comparable with the study conducted by A M Moorman et al, ${ }^{8}$ which had $60 \%$ as stroma low and $40 \%$ of cases as stroma high.

\begin{tabular}{|c|c|c|c|c|}
\hline $\begin{array}{c}\text { Sl. } \\
\text { No. }\end{array}$ & Study & Year & $\begin{array}{c}\text { No. of } \\
\text { Cases }\end{array}$ & Result \\
\hline 1 & A M Moorman et al & 2012 & 124 & $60 \%$ Stroma Low \\
\hline 2 & Present study & 2017 & 134 & $\begin{array}{c}66.4 \% \text { Stroma } \\
\text { Low }\end{array}$ \\
\hline \multicolumn{3}{|c|}{ Table 6. Comparison of Stromal Density of Carcinoma } \\
Breast
\end{tabular}

In the present study, majority of cases (81 cases and $60.4 \%$ ) were having grade II Bloom - Richardson grade. It was found that as the grade of the tumour increases, the stromal density in the tumour decreases. The results were comparable to the study conducted by Moorman A M et al in 2012, which showed stroma low in $13.5 \%$ of grade II tumours and $86.5 \%$ of stroma high tumour.

In the present study, stromal density was found to be increased with aggressive behaviour of the tumour. No statistically significant association was obtained between age, nodal status at diagnosis or stage of the tumour at diagnosis with stromal density. These findings were comparable to a study conducted by Ebru Sener et al. ${ }^{9}$

In the present study, $50 \%$ cases were of Luminal A type followed by $26.9 \%$ of cases to be of Luminal B type. In a study conducted by Marion Cortet et al ${ }^{10} 77.6 \%$ cases were Luminal A type followed by $7.3 \%$ Luminal B type. In another study conducted by Howlader et al,11 $73 \%$ cases were Luminal A type, $10 \%$ Luminal B type. The difference may be due to comparatively smaller sample size in the present study.

\begin{tabular}{|c|c|c|c|c|}
\hline $\begin{array}{c}\text { Sl. } \\
\text { No. }\end{array}$ & Study & Year & $\begin{array}{c}\text { Sample } \\
\text { Size }\end{array}$ & $\begin{array}{c}\text { Luminal A } \\
\text { Type }\end{array}$ \\
\hline 1 & Marion Cortet et al & 2018 & 12040 & $77.6 \%$ \\
\hline 2 & Howlader et al & 2014 & 57483 & $73 \%$ \\
\hline 3. & Present study & 2017 & 134 & $50 \%$ \\
\hline \multicolumn{3}{|c|}{ Table 7. Comparison of Molecular Subtyping of Cancers } \\
\hline
\end{tabular}

The association between stromal density and microvessel density was found to be statistically significant with a $p$ value of 0.042 . The present study showed that as the tumour grade increases, the microvessel density also increases. Compared to $64.7 \%$ cases with grade I tumour having low MVD, only $47.4 \%$ cases with grade III tumour had low MVD. In the study conducted by Divya Rani MLN et al ${ }^{12}$ showed that intra tumoural mean MVD was highest in grade III tumours suggesting that tumour angiogenesis correlates with tumour grade.

In the present study, Tumour associated macrophage infiltration was also found to be increasing as the tumour grade increased ( $\mathrm{p}=0.407)$. These results were comparable to the findings obtained from the study conducted by Gwak J M et $\mathrm{al}^{13}$ which showed that high levels of infiltration by macrophages with high histologic grade of the tumour ( $p$ $<0.001$ ). The difference in $\mathrm{p}$ value of present study and 
previous studies, despite the similar behaviour of TAMs in both studies compared to grade of the tumour may be due to very low number of grade 3 tumours in the present study.

In a study by Russel D L et al ${ }^{14}$ it was found that there is significant positive correlation between tumour angiogenesis and macrophage infiltration. In the present study, the association between MVD and TAM is found to be statistically significant with a p value of $0.022(<0.05)$, indicating that macrophage infiltration is an inducer of angiogenesis and both can be used as determinants of tumour behaviour.

\begin{tabular}{|c|c|c|c|c|}
\hline Sl. No. & Study & Year & Sample Size & p Value \\
\hline 1 & Russel D et al & 1996 & 101 & 0.037 \\
\hline 2 & Present Study & 2018 & 134 & 0.022 \\
\hline Table 8. Comparison of Microvessel Density with Tumour \\
Associated Macrophages in Breast Carcinoma \\
\hline
\end{tabular}

But in a minor population in the present study, low angiogenic tumours were seen to have high macrophage density and vice versa. This shows that macrophages themselves may not necessarily be a major angiogenic influence in all highly vascular tumours and also tumours with high TAM density and low MVD might not have yet developed increased vascularity because this type of study only represents a single point in evolution of cancer.

Also, it can be that the relation between the two may be just a coincidence. But both MVD and TAM seem to be important factors determining tumour behaviour and they appear to be related, and so can be targets for tailored therapy in future and even macrophage population of breast carcinoma could be used as a target for future antiangiogenic therapies.

\section{CONCLUSION}

$66.4 \%$ of the study population showed low stromal density $(<50 \%), 59 \%$ of cases showed low microvessel density and $50 \%$ of the cases showed low tumour associated macrophage density. Maximal stromal density, microvessel density and tumour associated macrophage density was found in the 41 50 years age group and both the extremes of ages showed reduced values. No statistically significant association was detected between age, tumour grade, stage of the tumour, lymph node metastasis, or molecular subtypes with stromal density, microvessel density and tumour associated macrophage density.

Statistically significant association was found between microvessel density with stromal density and tumour associated macrophages.

\section{ACKNOWLEDGMENT}

I express my sincere gratitude to Dr. Sankar S., Professor and Head of Department of Pathology, and my guide for the valuable guidance, motivation and help during this study and throughout my course.

I am also thankful to all my teachers in the Department of Pathology all the laboratory and non-teaching staff, Department of Community Medicine.

\section{REFERENCES}

[1] Consensus document for management of Breast cancer. Indian Council of Medical Research, New Delhi, 2016.

[2] Lester SC. The Breast. In: Kumar V, Abbas A, Aster J, eds. Robbins and Cotran Pathologic basis of diseases. Vol. 2. South East edn. Gurgaon: 2014: p. 1043-72.

[3] Rakha EA, Reis-Filho JS, Baehner F, et al. Breast cancer prognostic classification in the molecular era: the role of histological grade. Breast Cancer Research 2010;12(4):207.

[4] Lakhani SR. Breast carcinoma. In: Ellis IO, Schnitt SJ, eds. WHO classification of tumors of breast. Lyon: IARC Press 2012: p. 1-195.

[5] Li H, Fan X, Houghton J, et al. Tumour microenvironment: the role of tumour stroma in cancer. Journal of Cellular Biochemistry 2007;101(4):805-15.

[6] Holness CL, Simmons DL. Molecular cloning of CD68, a human macrophage marker related to lysosomal glycoproteins. Blood 1993;81(6):1607-13.

[7] Lau SK, Chu PG, Weiss LM. CD163: a specific marker of macrophages in paraffin-embedded tissue samples. American Journal of Clinical Pathology 2004;122(5):794-801.

[8] Moorman AM, Vink R, Heijmans HJ, et al. The prognostic value of tumour - stroma ratio in triple negative breast cancer. Eur J Surg Oncol 2012;38(4):307-13.

[9] Sener E, Sipal S, Gundogdu C. Comparison of microvessel density with prognostic factors in invasive ductal carcinomas of the breast. Turkish Journal of Pathology 2016;32(3):164-70.

[10] Kurian AW, Fish K, Shema SJ, et al. Lifetime risks of specific breast cancer subtypes among women in four racial/ethnic groups. Breast Cancer Research 2010;12(6):R99.

[11] Collaborative Group on Hormonal Factors in Breast Cancer. Menarche, menopause and breast cancer risk: individual participant meta-analysis, including 118964 women with breast cancer from 117 epidemiological studies. The Lancet Oncology 2012;13(11):1141-51.

[12] Divyarani MN, Hitendrakumar ML, Swaroopraj BV. Significance of micro vessel density in invasive breast carcinoma. Annals of Pathology and Laboratory Medicine 2018;5(3):A184-A7.

[13] Gwak JM, Jang MH, Kim DII, et al. Prognostic value of tumor-associated macrophages according to histologic locations and hormone receptor status in breast cancer. PLoS One 2015;10(4):e0125728.

[14] Russell DL, Claire EL, Ruth W, et al. Association of Macrophage infiltration with angiogenesis and prognosis in invasive breast carcinoma. Cancer Research 1996;56(20):4625-9. 